\title{
Locais de Reconexão na Técnica de Reablação após Isolamento das Veias Pulmonares com Criobalão em Pacientes com Fibrilação Atrial Paroxística
}

\author{
Reconnection Sites in Redo Ablation after Cryoballoon Pulmonary Vein Isolation in Patients with Paroxysmal \\ Atrial Fibrillation \\ Rogelio Robledo Nolasco, ${ }^{1}{ }^{(1)}$ Gerardo De Leon-Larios, ${ }^{1}$ David Eduardo Bazzini-Carranza, ${ }^{1}$ Elias Zavaleta, ${ }^{10}$ Omar \\ Calixto-Vargas ${ }^{1}$ \\ Centro Medico Nacional 20 de Noviembre - Hemodinamia y Electrofisiologua, ${ }^{1}$ Ciudad de México - México
}

\section{Resumo}

Fundamento: Na fibrilação atrial paroxística (FAP), o isolamento das veias pulmonares com criobalão (IVP-CB) tem eficácia semelhante à da ablação por radiofrequência (IVP-RF). Em procedimentos de reablação após IVP-RF, a reconexão das VPs é alta, ao passo que em pacientes com reablação após IVP-CB, as informações são escassas.

Objetivo: Determinar os locais de reconexão das VPs em pacientes que foram submetidos à reablação após IVP-CB inicial.

Métodos: Pacientes que foram submetidos a um procedimento de reablação de fibrilação atrial, após um IVP-CB inicial para FAP foram incluídos. O mapeamento eletroanatômico do AE foi utilizado. Um local de reconexão foi definido com a presença de uma voltagem de $0,3 \mathrm{mV}$ ou maior nas VPs e condução unidirecional ou bidirecional nas VPs durante o ritmo sinusal. Os locais de reconexão foram identificados por meio de corte paraesternal longitudinal e posteriormente ablacionados com radiofrequência.

Resultados: Dos 165 pacientes submetidos ao IVP inicial, 27 necessitaram reablações, dos quais 18 (66,6\%) eram do sexo masculino, com média de idade de $55+12,3$ anos. $O$ tempo de recorrência foi de 8,9+6,4 meses. A reconexão das VPs foi encontrada em $21(77,8 \%)$ pacientes. Houve um total de 132 lacunas de condução, seis por paciente, 3,6 por VP. Um número significativo de lacunas ocorreu na região ântero-superior da VP superior esquerda (VPSE) e nas regiões septal e inferior da VP superior direita (VPSD).

Conclusões: As VPs superiores apresentaram os locais de maior reconexão, principalmente na região anterior da VPSE e na região septal da VPSD. A razão por trás disso pode ser devido à maior espessura da parede atrial e à dificuldade em alcançar o contato de criobalão adequado.

Palavras-chave: Arritmias Cardíacas; Fibrilação Atrial; Técnicas de Ablação; Veias Pulmonares; Ondas de Rádio; Eletrocardiografia/métodos; Eletrocardiologia Ambulatorial; Taquicardia Atrial Ectópica; Holter.

\footnotetext{
Abstract

Background: In paroxysmal atrial fibrillation (PAF), pulmonary vein isolation using cryoballoon (CB-PVI) has similar efficacy as radiofrequency ablation (RF-PVI) has. In redo ablation procedures following RF-PVI, PV reconnection is high, whereas in patients with redo following CB-PVI, information is scarce.

Objective: To determine the sites of PV reconnection in patients who underwent redo ablation after initial CB-PVI.

Methods: Patients who underwent an AF redo procedure, following an initial CB-PVI for PAF were included. LA electroanatomic mapping was used. A reconnection site was defined as the presence of a voltage of $0.3 \mathrm{mV}$ or greater in the PV and unidirectional or bidirectional conduction in the PV during sinus rhythm. Reconnections sites were identified using a clock-face view description and were ablated with radiofrequency afterwards.

Results: Out of the 165 patients who underwent initial PVI, 27 required redo ablations, of which 18 (66.6\%) were males, with a mean age of $55+12.3$ years. The time of recurrence was $8.9+6.4$ months. PV reconnection was found in 21 (77.8\%) patients. There was a total of 132 conduction gaps, six per patient, 3.6 per PV. A significant number of gaps were in the anterosuperior region of the left superior PV (LSPV), and in the septal and inferior regions of the right superior PV (RSPV).
}

Correspondência: Rogelio Robledo Nolasco •

Centro Medico Nacional 20 de Noviembre - Hemodinamia y Electrofisiologua - Av. Félix Cuevas 540 Ciudad de México Ciudad de México 03100 - México E-mail: rogelio_robledo@hotmail.com

Artigo recebido em 27/07/2019, revisado em 05/05/2020, aceito em 16/06/2020

DOI: https://doi.org/10.36660/abc.20190503 
Conclusions: The upper PVs had the most reconnection sites, mostly at the anterior region of the LSPV and the septal region of the RSPV. The reason behind this may be due to greater atrial wall thickness, and difficulty in achieving adequate cryoballoon contact.

Keywords: Arrhythmias, Cardiac; Atrial Fibrillation, Pulmonary Veins; Radio Waves; Electrocardiography/methods; Electrocardiography, Ambulatory; Tachycardia, Ectopic Atrial; Holter.

Full texts in English - http://www.arquivosonline.com.br

\section{Introdução}

O isolamento das veias pulmonares (IVP) é o fundamento da terapia de ablação na fibrilação atrial (FA) paroxística e persistente. As técnicas mais comuns para obter IVP são a ablação com criobalão (CB) e a ablação por radiofrequência ponto a ponto, ambas com resultados semelhantes..$^{1,2} \mathrm{~A}$ recorrência de FA após o procedimento inicial de IVP considera-se como sendo mediada pela reconexão da veia pulmonar (VP), visto que $80 \%$ dos pacientes submetidos à reablação apresentam recuperação da condução da VP em pelo menos um local. ${ }^{3-5} \mathrm{Um}$ estudo para determinar a presença de reconexões das VPs em pacientes submetidos à reablação após IVP-CB inicial foi realizado. Além disso, descreveram-se os locais com maior probabilidade de recuperação da condução da VP.

\section{Métodos}

Pacientes sintomáticos com FA que apresentaram recorrência após um IVP com crioablação inicial, e que foram submetidos a um procedimento de reablação, foram incluídos no estudo. Os pacientes que receberam terapia inicial de crioablação inicial foram aqueles com FA resistente a fármacos com fração de ejeção do ventrículo esquerdo (FEVE) preservada, um diâmetro ântero-posterior (AP) do átrio esquerdo de $55 \mathrm{~mm}$ ou menos e nenhuma evidência de trombos no apêndice atrial esquerdo (AE) em um ecocardiograma transesofágico.

O procedimento foi realizado sob sedação consciente, e as veias femorais foram utilizadas como acesso venoso. Um cateter decapolar (Webster ${ }^{\circledR}$ Decapolar Catheter Deflectable) foi posicionado no seio coronário e uma punção transseptal atrial guiada por EIC foi realizada utilizando uma bainha 8F (Preface Braided Guiding Sheath) e uma agulha de Brockenbrough (Agulha Transseptak BRK). Uma bainha orientável 12F (FlexCath®sheath Medtronic, Minneapolis, $M N, E \cup A$ ) e um cateter de mapeamento circular (Achieve Medtronic) foram então inseridos no AE. Um cateter de balão de crioablação de $28 \mathrm{~mm}$ (Arctic Front, Cryocath ${ }^{\mathrm{TM}}$, Medtronic, CA, EUA ou Arctic Front Advance, Minneapolis, $M N$ ) foi utilizado para administrar a terapia de crioablação no ântero de cada veia pulmonar. A terapia de crioablação foi administrada por 180 a 300 segundos até que uma temperatura mínima de $-40^{\circ} \mathrm{C}$ fosse atingida e o IVP assegurado. A terapia foi considerada bem-sucedida se o bloqueio de entrada e saída da VP foram ambos alcançados. Durante a isolação da VP direita, o cateter quadripolar foi posicionado na veia cava superior para estimulação contínua do nervo frênico em ciclo de 1.800 ms e saída de 20 mA para evitar paralisia do hemidiafragma.
Durante o acompanhamento, foram prescritos eletrocardiograma e monitor de Holter de 24 horas (ou marca-passo) três meses após o procedimento e depois de seis meses adicionais. A medicação antiarrítmica foi suspensa após os primeiros três meses se nenhuma FA ou taquiarritmia atrial fosse identificada. A recorrência foi definida com a presença de FA ou outra taquicardia atrial, em tira de eletrocardiograma ou durante pelo menos 30 segundos em Holter após a primeira consulta de acompanhamento.

O procedimento de reablação foi realizado sob sedação consciente, por meio de acesso da veia femoral e apenas uma punção transseptal atrial. O anticoagulante não foi suspenso para a realização do procedimento de ablação. Sistema Carto $3 \circledR$ (Biosense Webster, Diamond Bar, CA, EUA) e um cateter multipolar (Pentaray Nav, BiosenseWebster) foram utilizados para construir mapas de tensão do AE e de cada VP, adotando $<0,3 \mathrm{mV}$ como valor de corte para tecido cicatricial e $>1,0 \mathrm{mV}$ para tecido normal. O isolamento da VP foi definido como a ausência de atividade elétrica dentro da VP durante o ritmo sinusal ou FA e/ou a presença de bloqueio de entrada e saída se o paciente estivesse em ritmo sinusal.

Os locais com lacunas de condução foram identificados por meio de corte paraesternal longitudinal, sendo considerados 12 locais diferentes. Uma vez localizados os locais de reconexão das VPs, eles foram ablados com radiofrequência utilizando um cateter de radiofrequência baseada em sensor de força de contato irrigado (ThermoCool SmartTouch, Biosense Webster). Além disso, eletrogramas fragmentados foram ativamente pesquisados e marcados, principalmente na parede posterior e teto do AE. Se o paciente estivesse em ritmo sinusal, a estimulação atrial a uma duração do ciclo de $170 \mathrm{~ms}$ era adotado para induzir FA a fim de buscar eletrogramas fragmentados, que eram posteriormente ablacionados. A ablação da parede anterior e rebordo foi feita a $40 \mathrm{~W}$, ao passo que na parede posterior foi a $25 \mathrm{~W}$, com temperatura limite de $45^{\circ} \mathrm{C}$. A taxa de infusão de 17 a $30 \mathrm{~mL} / \mathrm{min}$ de solução salina normal e uma força de pressão de 6-30g foram utilizadas.

Se o paciente estava em FA, a ablação foi considerada bemsucedida se houvesse uma queda de impedância de 8-10 ohms, uma diminuição na amplitude ou eliminação de eletrogramas atriais. Se o paciente estava em ritmo sinusal, o sucesso da ablação foi determinado pela perda da captura de estimulação no local da ablação. Por fim, linhas de ablação foram feitas no teto e istmo mitral, e se flutter atrial típico fosse detectado, a ablação do istmo cavotricuspídeo também era realizada.

\section{Análise estatística}

Este é um estudo observacional descritivo. A distribuição dos dados foi testada com o teste de normalidade Shapiro Wilk. As variáveis categóricas foram expressas em número total 
e porcentagens, ao passo que as variáveis com distribuição normal foram expressas como média e desvio padrão. $\mathrm{O}$ programa SPSS v.20 foi utilizado para análise de dados. Significância estatística foi considerada se $p<0,05$.

\section{Resultados}

\section{População do estudo}

De 2014 a 2018, 164 pacientes foram submetidos a IVP com a técnica de crioablação com balão, dos quais 27 apresentaram recorrência de FA e precisaram ser submetidos à reablação, após um acompanhamento de 10,7+7,2 meses. Desses pacientes, 18 (66,6\%) eram homens com idade média de 55 $\pm 12,3$ anos, escore CHA2DS2-VASc médio de 1,9 $\pm 1,6$ e FEVE média de $60,8 \pm 17,2 \%$. Características adicionais dos pacientes podem ser observadas na Tabela 1. Foi necessário consentimento informado e por escrito antes do procedimento de reablação de FA.

\section{Técnica de reablação e reconexão de VPs}

As características de procedimentos de reablação estão descritas na Tabela 2. O tempo para recorrência de FA foi de 8,8 8 8,2 meses. Dos 27 pacientes estudados, a recorrência de FA foi detectada em 17 (62,9\%) deles, por monitoramento de Holter, sete $(25,9 \%)$ por ECG e três $(11,1 \%)$ por monitoramento do marca-passo. Além disso, 18 (66,6\%) estavam em ritmo sinusal; os outros estavam em FA. Um local de reconexão foi definido com a presença de uma voltagem de $0,3 \mathrm{mV}$ ou maior na VP e condução unidirecional ou bidirecional na VP durante o ritmo sinusal.

Três acessos venais foram obtidos por veias femorais. Em todos os casos, foi realizada apenas uma punção transseptal

\section{Tabela 1 - Características dos pacientes}

\begin{tabular}{|c|c|}
\hline Homens & $18(66,6)$ \\
\hline Idade (anos) & $55 \pm 12,3$ \\
\hline Hipertensão & $15(55,5)$ \\
\hline Diabetes mellitus & $8(29,6)$ \\
\hline Insuficiência cardíaca & $1(3,7)$ \\
\hline Histórico de infarto & $1(3,7)$ \\
\hline Histórico de AVC (\%) & $3(11,1)$ \\
\hline Portador de marca-passo (\%) & $3(11,1)$ \\
\hline Duração da FA (meses) & $13,2 \pm 13,5$ \\
\hline CHA2DS-VASC & $1,9 \pm 1,6$ \\
\hline Quantidade de fármacos antiarrítmicos testados & $1,2 \pm 0,6$ \\
\hline FEVE & $60,8 \pm 17,2$ \\
\hline Diâmetro do AE (mm) & $40,2 \pm 8,0$ \\
\hline Tempo antes da recorrência (meses) & $8,9 \pm 6,4$ \\
\hline $\begin{array}{l}\text { Os dados são expressos em números (\%) } \\
\text { padrão. AVC: acidente vascular cerebral; FA: } \\
\text { átrio esquerdo; FEVE: fração de ejeção do ven } \\
\text { análise dos dados foi realizada com o program }\end{array}$ & $\begin{array}{l} \pm \text { desvio } \\
\text { atrial; } A E: \\
\text { querdo. } A \\
20 .\end{array}$ \\
\hline
\end{tabular}

Tabela 2 - Características do procedimento de reablação

\begin{tabular}{lc}
\hline Pacientes com reconexão de VP & $21(77,8)$ \\
\hline Tempo antes da recorrência (meses) & $10,5 \pm 6,5$ \\
\hline Número de veias por paciente & $1,6 \pm 0,4$ \\
\hline Número de lacunas por paciente & $6,0 \pm 0,5$ \\
\hline Número de lacunas por VP & $3,6 \pm 0,3$ \\
\hline Lacunas da veia pulmonar superior esquerda & $56(42,4)$ \\
\hline Lacunas da veia pulmonar inferior esquerda & $12(9,1)$ \\
\hline Lacunas da veia pulmonar superior direita & $35(26,5)$ \\
\hline Lacunas da veia pulmonar inferior direita & $29(21,9)$ \\
\hline Ablação adicional realizada & $4(14,8)$ \\
\hline Eletrogramas de complexos atriais fracionados & $3(11,1)$ \\
\hline Isolamento do istmo cavotricuspídeo & $130 \pm 17$ \\
\hline Tempo total do procedimento (minutos) & $8,5 \pm 1,7$ \\
\hline Tempo de fluoroscopia (min) & \\
\hline $\begin{array}{l}\text { Os dados são expressos em números (\%) ou média } \\
\text { padrão. A análise dos dados foi realizada com o programa SPSS }\end{array}$
\end{tabular}

guiada por ultrassom intracardíaco; primeiro, o cateter Pentaray foi introduzido para realizar um mapeamento de tensão e condução do AE e VP. 109 VPs foram identificadas nos 27 pacientes submetidos à reablação, dos quais 36 (33,0\%) tinham pelo menos um local de reconexão. 22 pacientes $(81,5 \%)$ apresentavam pelo menos uma VP com lacuna de

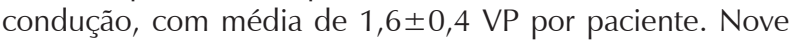
pacientes apresentaram um local de reconexão em uma VP (40,9\%), 11 pacientes (50\%) em duas VPs diferentes, um paciente $(4,5 \%)$ em três VPs e um paciente $(4,5 \%)$ em todas as quatro VPs.

\section{Localização das lacunas de condução em VP}

Um total de 132 lacunas de condução em IVP foi observado, com uma média de 6,0 0 , 5 lacunas de condução

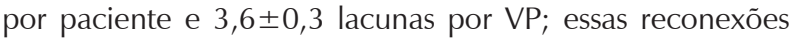
foram localizadas nos seguintes locais: $56(42,4 \%)$ na VP superior esquerda, 35 (26,5\%) na VP superior direita, 29 $(21,9 \%)$ na VP inferior direita e $12(9,1 \%)$ na VP inferior esquerda (Figura 1). O local com mais reconexões foi a junção da VPSE com o apêndice atrial esquerdo (rebordo endocárdico), seguido pela região posteroinferior da VPSE (71\%); e, por último, a região posterior da VPSE (29\%). A VPIE tinha menos lacunas de conexão, que foram distribuídas uniformemente ao redor da veia. A VPSD apresentou o maior número de reconexões fora das veias pulmonares direitas, principalmente nas regiões ântero-superior e inferior (94\% do total). As lacunas da VPID foram distribuídas uniformemente ao redor da veia, favorecendo levemente as regiões inferiores.

\section{Localizações fragmentadas de eletrogramas e outras arritmias}

Eletrogramas atriais fragmentados foram identificados em oito $(29,6 \%)$ pacientes, principalmente na parede posterior. 


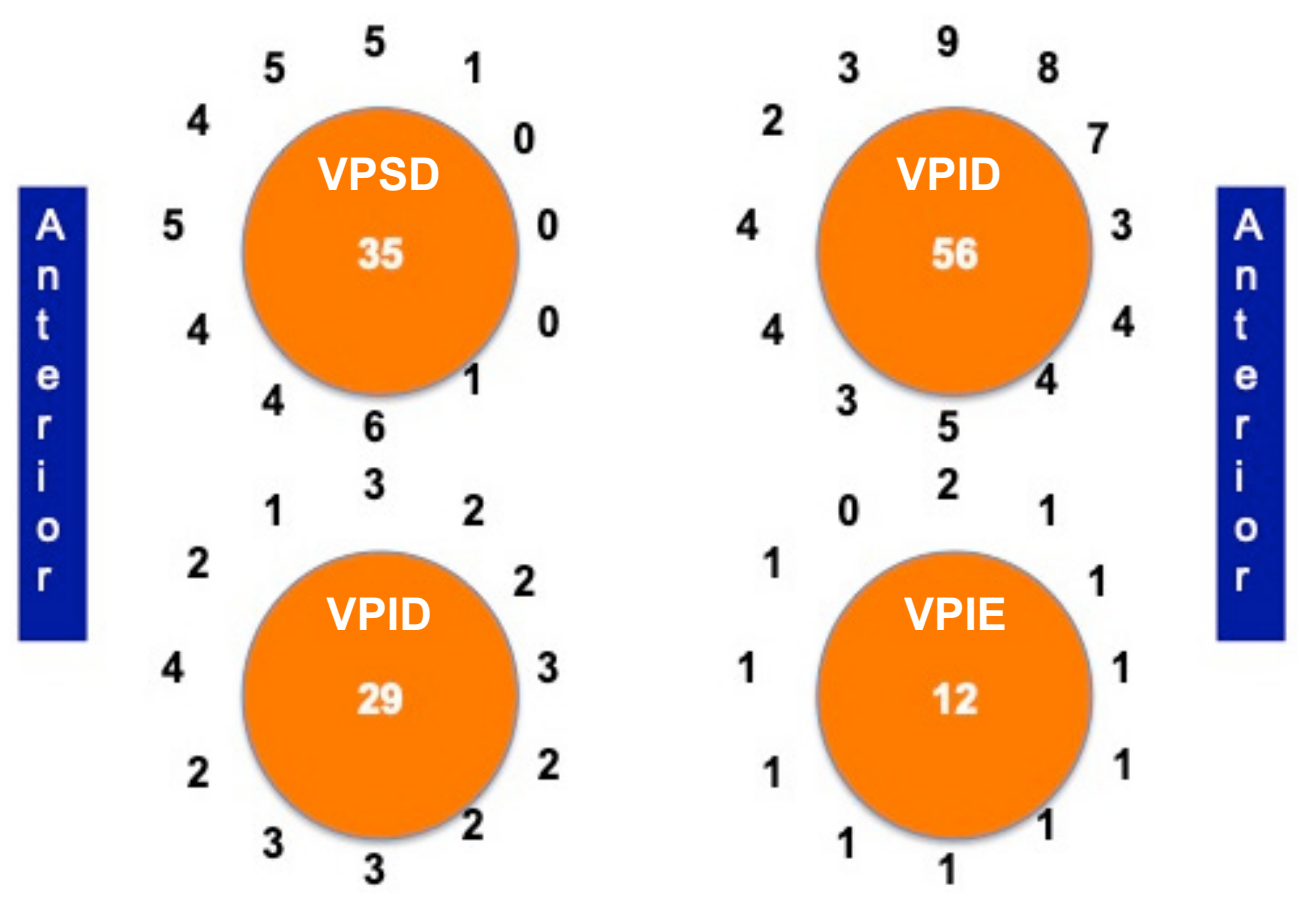

Figura 1 - Distribuição das lacunas de reconexão nas quatro VPs (o número no centro de cada círculo). A VPSE é a mais reconectada, 71\% das lacunas ocorrem nas regiões ântero-superior e ântero-inferior. A VPSD teve mais reconexões nas regiões ântero-superior e septal. VPIE: veia pulmonar inferior esquerda; VPSE: veia pulmonar superior esquerda; VPID: veia pulmonar inferior direita; VPSD: veia pulmonar superior direita.

O flutter atrial típico foi encontrado em nove (33,3\%) pacientes, submetidos à ablação do istmo cavo-tricuspídeo até a obtenção do bloqueio bidirecional.

A duração média dos procedimentos de reablação de RF foi de 130 \pm 17 minutos, com um tempo médio de fluoroscopia de $8,5 \pm 1,7$ minutos. Ocorreram duas complicações relacionadas ao procedimento, um hematoma inguinal submetido a tratamento conservador e um derrame pericárdico que foi prontamente resolvido após a punção pericárdica.

\section{Discussão}

O isolamento da veia pulmonar (IVP) por radiofrequência (RF) ponto a ponto demonstrou ser um tratamento eficaz para FA paroxística e, como tal, é atualmente recomendado nas diretrizes clínicas de FA. ${ }^{6} \mathrm{O}$ fundamento da terapia de ablação de FA paroxística é o IVP, que tradicionalmente tem sido realizado com radiofrequência. ${ }^{7-9}$ Mais recentemente, a crioablação surgiu como alternativa viável, com resultados semelhantes. ${ }^{1,2}$ No estudo de Fogo e Gelo, ambas as abordagens foram igualmente eficazes, especialmente ao comparar o criobalão de segunda geração com o cateter de força de contato. ${ }^{10}$

Em pacientes submetidos a um procedimento de reablação após um IVP inicial, lacunas na conexão das VPs podem ser encontradas em mais de $95 \%$ das vezes. As veias pulmonares esquerdas parecem ter mais reconexões, especialmente nas áreas anterior e inferior. ${ }^{4,11,12}$ Katering et al., ${ }^{13}$ publicaram uma série de casos de procedimentos de reablação de FA, documentando uma média de 2,9 reconexões das VPs em comparação com nossos achados, 1,6. Recentemente, outro estudo publicado mostrou um tempo de recorrência após IVP com crioablação e radiofrequência de 7,4 48,8 meses e $9,8 \pm 14,5$ meses, respectivamente, o que foi semelhante aos

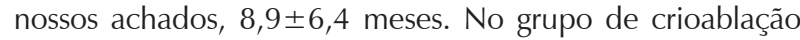
desse estudo, 80,6\% apresentavam pelo menos uma VP com lacunas de condução, com média de 2,9 lacunas por VP, que se distribuíram igualmente entre as quatro VPs. Em nosso estudo, $81,5 \%$ dos pacientes apresentavam pelo menos uma lacuna de condução de VP, com média de 6,0 lacunas por paciente e 3,6 gaps por VP. A veia com mais lacunas de condução foi a VPSE, seguida da VPSD, VPIE e VPID. A razão por trás de nossos números de lacunas mais altos pode ser explicada pela maneira como os medimos. Identificamos 12 regiões diferentes nas VPs, em oposição às oito utilizadas por outros autores. Em outros dois estudos, reconexões de VPs foram encontradas em 54 e 71\% dos pacientes, e a região anterolateral da VPSE (onde a crista endocárdica pode ser encontrada) foi o local mais frequente de lacunas de condução. ${ }^{14,15}$ De maneira geral, a região ântero-superior das veias superiores foi o local com mais lacunas, nos estudos citados e no presente estudo. Entretanto, ao passo que a VPID foi a VP inferior com mais reconexões, nossos resultados mostraram a região ínfero-anterior como a principal fonte de reconexões, o que difere dos achados de Katering et al., ${ }^{13}$ que observaram uma distribuição mais homogênea. Por fim, eletrogramas fracionados no AE foram encontrados em 29,6\% dos pacientes, incidência muito superior à relatada por Galand et al. ${ }^{5}$ 
Acreditamos que nos locais em que há mais reconexões, o criobalão não tem contato adequado e, portanto, a crioterapia não atinge uma ablação mais profunda. Esse fenômeno ocorre porque o formato do ântero da VP nem sempre é circular, muitas vezes é ovalada, e as dimensões de cada veia também variam, portanto, o suporte do criobalão não é homogêneo. ${ }^{14}$ Além disso, uma lesão de crioablação adequada deve ter profundidade suficiente. Visto isso, locais do ântero da VP com parede mais espessa, como a junção da VPSE com o óstio do apêndice do AE (rebordo endocárdico), estão sujeitos a lesões não transmurais. Kowalski et al. confirmaram essa suposição ao mostrar em corações humanos dissecados que as lacunas de condução da VP ocorriam quando a lesão de radiofrequência não era transmural. ${ }^{16}$ Finalmente, uma técnica adequada é importante para obter a oclusão da veia pulmonar antes de administrar a terapia de crioablação. Diversas estratégias diferentes foram descritas. No entanto, sempre haverá casos em que o isolamento completo é impossível. ${ }^{17}$

Uma limitação do presente estudo reside no seu caráter descritivo. Um projeto prospectivo longitudinal forneceria mais resultados clinicamente relevantes.

\section{Conclusão}

A incidência de lacunas na condução das VP no presente estudo foi semelhante aos achados de outros estudos. As veias pulmonares superiores apresentam a maioria das lacunas de condução e estão na região ântero-superior no apêndice da AE e na junção da VPSE, e em direção à parte do septo na VPSD. A falta de contato adequado do balão devido a variações anatômicas na VP, técnica inadequada e locais com

\section{Referências}

1. Kuck KH, Brugada J, Furnkranz A, Metzner A, Ouyang F, Chun KR, et al, for the FIRE AND ICE Investigators. Cryoballoon or radiofrequency ablation for paroxysmal atrial fibrillation. N Engl J Med 2016;374(23):2235-45.

2. Buiatti A, von Olshausen G, Barthel P, Schneider S, Luik A, Kaess B, et al. Cryoballoon vs. radiofrequency ablation for paroxysmal atrial fibrillation: an updated metaanalysis of randomized and observational studies. Europace 2017; 19(3):378-84.

3. Rajappan K, Kistler PM, Earley MJ, Thomas G, Izquierdo M, Sporton SC, et al. Acute and chronic pulmonary vein reconnection after atrial fibrillation ablation: a prospective characterization of anatomical sites. Pacing Clin Electrophysiol. 2008; 31(12):1598-605.

4. Ouyang F, Antz M, Ernst S, Hachiya H, Mavrakis H, Deger FT, et al. Recovered pulmonary vein conduction as a dominant factor for recurrent atrial tachyarrhythmias after complete circular isolation of the pulmonary veins: lessons from double Lasso technique. Circulation. 2005 Jan 18;111(2):127-35.

5. Galand V, Pavin D, Behar N, Auffret V, Fénéon D, Behaghel A et al. Localization of gaps during redo ablations of paroxismal atrial fibrillation: Preferential patterns depending on the choice of cryoballoon ablation or radiofrequency ablation for the initial procedure. Arch Cardiovasc Dis. 2016;109(11):591-8.

6. $2017 \mathrm{HRS} / \mathrm{EHRA} / \mathrm{ECAS} / \mathrm{APHRS} / \mathrm{SOLAECE}$ expert consensus statement on catheter and surgical ablation of atrial fibrillation. Europace 2017; 00: $1-160$. parede espessa no ântero da VP são a base das reconexões após o procedimento inicial de ablação de FA com criobalão. Finalmente, em pacientes com FA a recorrência após IVP-CB inicial, cerca de $30 \%$ têm gatilhos, que estão no corpo do $\mathrm{AE}$, principalmente na parede posterior.

\section{Contribuiç̧ão dos autores}

Concepção e desenho da pesquisa e Análise e interpretação dos dados: Nolasco RR; Obtenção de dados: Bazzini-Carranza DE, Zavaleta E; Redação do manuscrito: Nolasco RR, LeonLarios GD; Revisão crítica do manuscrito quanto ao conteúdo intelectual importante: Leon-Larios GD, Calixto-Vargas O.

\section{Potencial conflito de interesse}

Não há conflito com o presente artigo

\section{Fontes de financiamento}

O presente estudo não teve fontes de financiamento externas.

\section{Vinculação acadêmica}

Não há vinculação deste estudo a programas de pósgraduação.

\section{Aprovação Ética e Consentimento Informado}

Este artigo não contém estudos com humanos ou animais realizados por nenhum dos autores.

7. Pappone C, Rosanio S, Oreto G, Tocchi M, Gugliotta F, Vicedomini G, et al. Circumferential radiofrequency ablation of pulmonary vein ostia: A new anatomic approach for curing atrial fibrillation. Circulation 2000; 102(21):2619-2628.

8. Ouyang F, Tilz R, Chun J, Schmidt B, Wissner E, Zerm T, et al. Long-term results of catheter ablation in paroxysmal atrial fibrillation: lessons from a 5-year follow-up. Circulation 2010;122(23):2368-2377.

9. Weerasooriya R, Khairy P, Litalien J, Macle L, Hocini M, Sacher F, et al. Catheter ablation for atrial fibrillation: are results maintained at 5 years of follow-up? J Am Coll Cardiol 2011;57(2):160-166.

10. Kuck K, Brugada J, Schluter M, Braegelmann KM, Kueffer FJ, Chun J, et al. The FIRE AND ICE Trial: What We Know, What We Can Still Learn, and What We Need to Address in the Future. J Am Heart Assoc. 2018;7:e010777.

11. Mainigi SK, Sauer WH, Cooper JM, Dixit S, Gerstenfeld EP, Callans DJ, et al. Incidence and predictors of very late recurrence of atrial fibrillation after ablation. J Cardiovasc Electrophysiol .2007;18(1):69-74.

12. Van der zee SA, D'avila A. Redo Procedures in Patients with Paroxysmal Atrial Fibrillation. J Innov Cardiac Rhytm Manag. 2010;1:44-52.

13. Kettering K, Gramley F. Radiofrequency catheter ablation for redo procedures after pulmonary vein isolation with the cryoballoon technique. Long-term outcome. Herzschr Elektrophys. .2017;28(2):225-31.

14. Godin B, Savoure A, Gardey K, Anselme F. Lessons fromradiofrequency redo procedure after cryoballoon pulmonaryvein isolation for paroxysmal atrial fibrillation. Circ J.2013;77:2009-13. Doi:10.1253/circj-cj-13-0046. 
15. Furnkranz A, Chun KR, Nuyens D, Metzner A, Koter I, Schmidt B, et al. Characteriza-tion of conduction recovery after pulmonary vein isolationusing the "single big cryoballoon" technique. Heart Rhythm2010;7(2):184—90.

16. Kowalski M, Grimes MM, Perez FJ, Kenigsberg DN, Koneru J, Kasirajan V, et al. Histopathologic characterization of chronic radiofrequency ablation lesions for pulmonary vein isolation. J Am Coll Cardiol. 2012; 59(10):930-8
17. Chun KR, Schmidt B, Metzner A, Tilz R, Zerm T, Köster I, et al. The 'single big cryoballoon' technique for acute pulmonary vein isolation in patients with paroxysmal atrial fibrillation: a prospective observational single centre study. Eur Heart J 2009;30(6):699-709. 